\title{
Um estudo sobre a relação entre os elementos de jogos e os tipos de usuários de sistemas gamificados
}

\author{
Rafaela Melo \\ Marcela Pessoa \\ Universidade do Estado do Amazonas \\ Manaus, Amazonas, Brasil \\ \{rmf.lic16,msppessoa\}@uea.edu.br
}

\author{
Gabriel Haydar \\ David Fernandes Oliveira \\ Elaine H. T. de Oliveira \\ Leandro Galvão de Carvalho \\ Tayana Conte \\ Universidade Federal do Amazonas \\ Manaus, Amazonas, Brasil \\ \{gigh,david,elaine,galvao,tayana\}@icomp.ufam.edu.br
}

\author{
Filipe Dwan Pereira \\ Universidade Federal de Roraima \\ Boa Vista, Roraima, Brasil \\ filipe.dwan@ufrr.br
}

Gamificação, ou gamification, é definida como a utilização de elementos de jogos em contextos não relacionados a jogos, como economia, saúde e educação, com o intuito de deixar as atividades mais divertidas e motivadoras [8]. Na área de educação, é amplamente utilizada para gerar engajamento dos alunos e aumentar a motivação para o aprendizado [3,16]. Segundo [1], essa ampla utilização deve-se ao fato de que muitas pessoas em idade escolar fazem uso constante de jogos, e que, portanto incorporar elementos de jogos como desafios, recompensas e rankings dentro das atividades de sala de aula pode aumentar o engajamento nas disciplinas.

Muitos estudos $[6,7,9,11,20,21]$ têm demonstrado que, em ambientes gamificados, cada usuário é motivado de uma forma diferente. Dessa forma, determinados elementos de jogos podem ser motivadores para alguns usuários, mas para outros não, o que pode impactar o desempenho de cada indivíduo dentro das atividades propostas. Diante disso, vê-se a importância de classificar os usuários de acordo com suas características pessoais e, a partir disso, propor elementos específicos para cada tipo de usuário, com o intuito de tornar a experiência mais satisfatória para todos [4].

$\mathrm{Na}$ literatura, existem várias propostas de classificação de usuários para jogos ou sistemas gamificados como as tipologias de Bartle [2] e BrainHex [13], o modelo HEXAD [12], entre outras. Nessas propostas, os usuários são classificados de acordo com suas preferências e tendências pessoais, identificadas através de questionários. O modelo HEXAD, por exemplo, classifica os usuários de sistemas gamificados em seis tipos: filantropo, socializador, espírito livre, conquistador, jogador e disruptor. Segundo esses trabalhos, cada tipo de usuário é motivado por diferentes aspectos dos jogos. Porém, apesar de existirem várias tipologias de usuários para jogos ou ambientes gamificados, a literatura [10,19] ainda carece de estudos mais aprofundados sobre quais elementos de jogos mais motivam e despertam o engajamento de cada tipo de usuário, bem como quais as tendências e comportamentos de cada tipo dentro de um ambiente gamificado. Vale ressaltar que esse conhecimento é valioso para os designers de sistemas gamificados, pois sem ele é desafiador criar sistemas que sejam realmente capazes de motivar e engajar os diferentes tipos de usuários [14]. Diante disso, no presente trabalho

Fica permitido ao(s) autor(es) ou a terceiros a reprodução ou distribuição, em parte ou no todo, do material extraído dessa obra, de forma verbatim, adaptada ou remixada, bem como a criação ou produção a partir do conteúdo dessa obra, para fins não comerciais, desde que sejam atribuídos os devidos créditos à criação original, sob os termos da licença CC BY-NC 4.0.

EduComp'21, Abril 26-30, 2021, Jataí, Goiás, Brasil (On-line)

(C) 2021 Copyright mantido pelo(s) autor(es). Direitos de publicação licenciados à Sociedade Brasileira de Computação (SBC). está sendo conduzido um estudo sobre cada tipo de usuário através de uma plataforma de gamificação chamada CodePlay [15].

O CodePlay é uma plataforma de gamificação baseada em elementos de jogos RPG [17, 18, 22], implementada dentro do Instituto de Computação (IComp) da Universidade Federal do Amazonas (UFAM), e integrada ao juiz on-line CodeBench ${ }^{1}[5]$. Ao solucionar um exercício de programação no juiz on-line, uma carta é sorteada para o aluno, definindo sua sorte e permitindo que ele avance na estória do jogo. Dentro do ambiente (mundo do jogo), os alunos podem interagir com colegas, coletar recompensas, realizar missões, vencer inimigos e explorar vários elementos existentes no mapa.

Para atender aos diferentes tipos de usuários, que serão identificados por meio do modelo HEXAD [12, 21], foram adicionados novos elementos de jogos ao CodePlay, dentre os quais destacam-se: (i) Doações de itens e armas: permite a doação de itens e armas entre alunos; (ii) Trocas de mensagens: possibilita a troca de mensagens entre alunos; (iii) Itens de destaque de mérito: permite que os alunos colecionem medalhas que atestam conquistas relacionadas à disciplina; (iv) Barra de progresso: são indicações visuais dos lugares fechados, passagens secretas e easter eggs já encontrados pelos usuários em cada fase. Nelas é possível acompanhar as conquistas do aluno no jogo e no juiz on-line, como média nas provas e nas listas de exercícios; (v) Ranking com rating dos jogadores: os jogadores são divididos em três grupos, de acordo com o valor de experiência (XP) de cada um: Bronze, Prata ou Ouro; (vi) Missões (quests): a cada fase do jogo é disponibilizada uma missão e, em algumas delas, o aluno precisa lutar contra monstros; (vii) Lugares secretos: assim como as missões, todas as fases contêm lugares secretos, onde os alunos podem se deparar com outros personagens, encontrar moedas e força, armas e itens; e (viii) Easter Eggs: são dispostos dentro de lugares fechados e de forma aleatória nas fases a fim de manter a imprevisibilidade.

Após a implementação dos novos elementos e por ser uma plataforma de gamificação baseada em RPG, o CodePlay possui atualmente uma quantidade grande e diversificada de elementos de jogos, tornando um ambiente bastante propício para um estudo detalhado sobre as preferências, comportamentos e o que motiva cada tipo de usuário. Será possível, ainda, analisar o quanto cada elemento de jogo tem potencial para engajar os diferentes tipos de usuários/jogadores.

\footnotetext{
${ }^{1} \mathrm{http} / / /$ codebench.icomp.ufam.edu.br
} 


\section{AGRADECIMENTOS}

Esta pesquisa, realizada no âmbito do Projeto Samsung-UFAM de Ensino e Pesquisa (SUPER), nos termos do artigo 48 do Decreto $\mathrm{n}^{\circ}$ 6.008/2006 (SUFRAMA), foi parcialmente financiada pela Samsung Eletrônica da Amazônia Ltda., nos termos da Lei Federal $\mathrm{n}^{\circ}$ 8.387/1991, por meio dos convênios 001/2020 e 003/2019, firmados com a Universidade Federal do Amazonas e a FAEPI, Brasil, além do apoio da Coordenação de Aperfeiçoamento de Pessoal de Nível Superior - Brasil (CAPES) - Código de Financiamento 001 e do Conselho Nacional de Desenvolvimento Científico e Tecnológico Brasil (Processo 308513/2020-7).

\section{REFERÊNCIAS}

[1] Brian J Arnold. 2014. Gamification in education. Proceedings of the American Society of Business and Behavioral Sciences 21, 1 (2014), 32-39.

[2] Richard Bartle. 1996. Hearts, clubs, diamonds, spades: Players who suit MUDs. Journal of MUD research 1, 1 (1996), 19.

[3] Felipe Cechella, Gardênia Abbad, and Ralf Wagner. 2021. Leveraging learning with gamification: An experimental case study with bank managers. Computers in Human Behavior Reports 3 (2021), 100044. https://doi.org/10.1016/j.chbr.2020. 100044

[4] Lamya F Daghestani, Lamiaa F Ibrahim, Reem S Al-Towirgi, and Hesham A Salman. 2020. Adapting gamified learning systems using educational data mining techniques. Computer Applications in Engineering Education 28, 3 (2020), 568-589.

[5] Leandro Galvão, David Fernandes, and Bruno Gadelha. 2016. Juiz online como ferramenta de apoio a uma metodologia de ensino híbrido em programação. In Brazilian Symposium on Computers in Education (SBIE), Vol. 27. 140.

[6] Muhammad Awais Hassan, Ume Habiba, Fiaz Majeed, and Muhammad Shoaib. 2019. Adaptive gamification in e-learning based on students' learning styles. Interactive Learning Environments (2019), 1-21.

[7] Yuan Jia, Bin Xu, Yamini Karanam, and Stephen Voida. 2016. Personality-targeted gamification: a survey study on personality traits and motivational affordances. In Proceedings of the 2016 CHI Conference on Human Factors in Computing Systems. 2001-2013.

[8] Karl M Kapp. 2012. The gamification of learning and instruction: game-based methods and strategies for training and education. John Wiley \& Sons.

[9] Ana Carolina Tomé Klock, Isabela Gasparini, Marcelo Soares Pimenta, and Juho Hamari. 2020. Tailored gamification: A review of literature. International Journal of Human-Computer Studies (2020), 102495.

[10] Elise Lavoué, Baptiste Monterrat, Michel Desmarais, and Sébastien George. 2018 Adaptive gamification for learning environments. IEEE Transactions on Learning Technologies 12, 1 (2018), 16-28.

[11] Christian E Lopez and Conrad S Tucker. 2019. The effects of player type on performance: A gamification case study. Computers in Human Behavior 91 (2019), 333-345.

[12] Andrzej Marczewski. 2015. Even Ninja Monkeys like to play. CreateSpace Indep. Publish Platform, Charleston, Chapter User Types (2015), 69-84.

[13] Lennart E Nacke, Chris Bateman, and Regan L Mandryk. 2014. BrainHex: A neurobiological gamer typology survey. Entertainment computing 5, 1 (2014), $55-62$.

[14] Rita Orji, Gustavo F Tondello, and Lennart E Nacke. 2018. Personalizing persuasive strategies in gameful systems to gamification user types. In Proceedings of the 2018 CHI Conference on Human Factors in Computing Systems. 1-14.

[15] Marcela Pessoa, David Fernandes, Leandro Silva Galvão de Carvalho, Elaine Oliveira, Walter Nakamura, and Tayana Conte. 2019. CodePlay: Uma Plataforma de Gamificação baseada em Jogos de RPG Multiplayer. In Brazilian Symposium on Computers in Education (Simpósio Brasileiro de Informática na Educação-SBIE), Vol. 30. 843 .

[16] Ralph Breno Silva Ribeiro, Leandro Silva Galvão de Carvalho, Elaine Harada Teixeira de Oliveira, David Braga Fernandes de Oliveira, and Marcela Savia Picanco Pessoa. 2020. Investigação empírica sobre os efeitos da gamificação de um juiz online em uma disciplina de introdução à programação. Revista Brasileira de Informática na Educação 28 (2020), 461-490.

[17] Benjamin Sanders and Jims Marchang. 2016. A novel taxonomy of opportunities and risks in massively multiplayer online role playing games. In Proceedings of the 8th International Conference on Management of Digital EcoSystems. 90-98. https://doi.org/10.1145/3012071.3012094

[18] Anne Sullivan, Michael Mateas, and Noah Wardrip-Fruin. 2010. Rules of Engagement: Moving Beyond Combat-based Quests. In Proceedings of the Intelligent Narrative Technologies III Workshop (Monterey, California) (INT3 '10). ACM, New York, NY, USA, Article 11, 8 pages. https://doi.org/10.1145/1822309.1822320

[19] Armando Toda, Filipe Dwan Pereira, Ana Carolina Tomé Klock, Luiz Rodrigues, Paula Palomino, Wilk Oliveira, Elaine Harada Teixeira Oliveira, Isabela Gasparini, Alexandra Ioana Cristea, and Seiji Isotani. 2020. For whom should we gamify? Insights on the users intentions and context towards gamification in education. In Anais do XXXI Simpósio Brasileiro de Informática na Educação. SBC, 471-480.
[20] Gustavo F Tondello, Karina Arrambide, Giovanni Ribeiro, Andrew Jian-lan Cen, and Lennart E Nacke. 2019. "I don't fit into a single type": A Trait Model and Scale of Game Playing Preferences. In IFIP Conference on Human-Computer Interaction. Springer, 375-395.

[21] Gustavo F Tondello, Rina R Wehbe, Lisa Diamond, Marc Busch, Andrzej Marczewski, and Lennart E Nacke. 2016. The gamification user types hexad scale. In Proceedings of the 2016 annual symposium on computer-human interaction in play. 229-243.

[22] Anders Tychsen, Michael Hitchens, and Thea Brolund. 2008. Motivations for Play in Computer Role-playing Games. In Proceedings of the 2008 Conference on Future Play: Research, Play, Share (Toronto, Ontario, Canada) (Future Play '08). ACM, New York, NY, USA, 57-64. https://doi.org/10.1145/1496984.1496995 